\title{
INVERSIÓN Y FINANCIARIZACIÓN: UN MODELO MATEMÁTICO KALECKIANO
}

\section{Faustino Vega Miranda*}

\section{Resumen}

Uno de los temas que ha adquirido importancia en fechas recientes es la financiarización y su relación con la inversión, por lo que el presente trabajo realiza una revisión de modelos matemáticos que explican la dinámica de la inversión. Con el objetivo de lograr una mejor comprensión del tema, se estudia una propuesta keynesiana convencional, un modelo kaleckiano y un modelo que formaliza la financiarización.

\section{Abstract}

One of the issues that has recently become important is financialization and its relationship with investment, which is why this paper reviews mathematical models that explain the dynamics of investment. In order to achieve a better understanding of the subject, we study a conventional Keynesian proposal, a Kaleckian model and a model that formalizes financialization.

Palabras clave: macroeconomía, modelo matemático, financiarización, inversión productiva Keywords: macroeconomics, mathematical model, financialization, productive investment Clasificación JEL: E00, C02, E22, G31

* Profesor adscrito a la Facultad de Estudios Superiores Acatlán de la Universidad Nacional Autónoma de México (UNAM), correo electrónico: <kopola9@yahoo.com.mx >. El autor agradece los oportunos comentarios de Lidia Vega Miranda, así como el apoyo técnico de los dictaminadores anónimos de esta revista. 


\section{Introducción}

En la ciencia económica, teoría y matemáticas suscitan controversias en cuanto al enfoque metodológico y lleva a posicionamientos con tintes, en ocasiones, más ideológicos que objetivos. Entonces, ¿cuál es la pertinencia de teorizar sobre la base de modelos matemáticos?

En las ciencias naturales existe una pared invisible que separa el objeto y el sujeto de estudio, esa muralla es conocida como objetividad. El biólogo tiene la posibilidad de reunir los reactivos A-B y siempre llegar al reactivo C. No hay posibilidad de la intromisión de las filias y las fobias personales.

En las ciencias sociales, específicamente en Economía, es complicada la dinámica de la investigación por la imposibilidad de experimentos controlados como en las ciencias naturales. Un economista no puede recrear una crisis económica, sin embargo, para compensar la discapacidad de nuestra ciencia existen las interpretaciones, los prejuicios, las doctrinas, las teorías y los modelos.

Los economistas usan modelos matemáticos porque aportan rigor, favorecen la comprensión de las ideas expuestas y ayudan a establecer las recomendaciones de política económica. Debido a esto, es necesario retomar las matemáticas desde una postura heterodoxa para explicar los viejos problemas económicos como la determinación de la inversión productiva desde la visión keynesiana y kaleckiana. Además, esta postura permite explicar los nuevos procesos capitalistas como la financiarización.

De esta forma, el objetivo del presente ensayo es proponer un modelo matemático que analice el fenómeno económico llamado financiarización. El artículo se compone de tres secciones, en la primera parte se estudian dos propuestas con características keynesianas bajo el enfoque del modelo IS-LM; la segunda sección aborda una propuesta matemática de las ideas de Kalecki sobre la inversión productiva. Y, en la tercera parte, se deriva un modelo con elementos kaleckianos sobre la base del fenómeno que define el capitalismo contemporáneo, de acuerdo a la visión heterodoxa: la financiarización. El documento finaliza cuando se derivan algunas conclusiones.

\section{Dos modelos keynesianos}

Las ideas de Keynes, contenidas en La teoría general, se formalizaron en el marco del modelo IS-LM por Hicks en 1937, donde el instrumento matemático se concibió como una propuesta generalizadora entre las ideas clásicas y keynesianas. En ese sentido, es esta sección del ensayo se retoman dos formas del modelo desde la perspectiva del mercado de bienes excluyendo el dinero porque esta investigación no busca ahondar en dicha temática. Por un lado, en la versión A el sistema de ecuaciones se indeterminada y en la versión B la inversión es una variable exógena. Por tanto, en los dos modelos los supuestos básicos con los siguientes: existe 
una economía cerrada, no hay gobierno y es una economía del tipo competencia perfecta.

En la primera propuesta se establecen 3 ecuaciones. En la primera ecuación el ingreso $(Y)$ es la suma de consumo $(C)$ y de inversión $(I)$. En la segunda ecuación el consumo está explicado por una parte autónoma $(\mathrm{Co})$ y por otra parte que depende del ingreso $(\mathrm{cY})$. Keynes (2014) definió que la propensión marginal a consumir $(c)$ es positiva y menor que la unidad. $\mathrm{Y}$, finalmente, la tercera ecuación explica que la inversión está determinada por la tasa de interés $(i)$, ya que entre las dos variables existe una relación inversa.

$$
\begin{gathered}
Y=C+I \\
C=C o+c Y
\end{gathered}
$$

$$
I=I o-b i
$$

El sistema de ecuaciones se puede escribir en notación matricial con la forma $A X=d$, donde $A$ representa el conjunto de coeficientes, $x$ es el conjunto de variables y $d$ es el conjunto de términos constantes.

$$
\left[\begin{array}{llll}
1 & -1 & -1 & 0 \\
-c & 1 & 0 & 0 \\
0 & 0 & 1 & b
\end{array}\right]\left[\begin{array}{c}
Y \\
C \\
I \\
i
\end{array}\right]=\left[\begin{array}{c}
0 \\
C_{0} \\
I_{0}
\end{array}\right]
$$

Sin embargo, las ecuaciones son inconsistentes y no se puede calcular una solución. Desde otra óptica, la matriz $A$ no es cuadrada por lo que no se puede calcular el determinante. Una solución a este problema es reformular la tercera ecuación, es decir, la inversión está definida desde fuera del modelo, es exógena. Aunque pierde realismo y capacidad de explicación la propuesta teórica.

$$
\begin{gathered}
Y=C+I \\
C=C o+c Y \\
I=I o
\end{gathered}
$$

Se puede establecer el sistema de ecuaciones, donde el determinante de la matriz $A$ es diferente de cero y asegura una solución trivial. Dicho determinante es renombrado como el parámetro $s$ o propensión marginal a ahorrar.

$$
\left[\begin{array}{ccc}
1 & -1 & -1 \\
-c & 1 & 0 \\
0 & 0 & 1
\end{array}\right]\left[\begin{array}{c}
Y \\
C \\
I
\end{array}\right]=\left[\begin{array}{c}
0 \\
C o \\
I o
\end{array}\right]
$$




$$
|A|=1\left[\begin{array}{cc}
1 & -1 \\
-c & 1
\end{array}\right]=1-c=s
$$

El producto de equilibrio $\left(Y^{*}\right)$, el consumo de equilibrio $\left(C^{*}\right)$ y la inversión de equilibrio $\left(I^{*}\right)$ son mayores a cero, esta última variable está definida exógenamente.

$$
Y^{*}=\frac{\left[\begin{array}{ccc}
0 & -1 & -1 \\
C o & 1 & 0 \\
I o & 0 & 1
\end{array}\right]}{s}=\frac{I o}{s}
$$

$$
\begin{gathered}
C^{*}=\frac{\left[\begin{array}{ccc}
1 & 0 & -1 \\
-c & C o & 0 \\
0 & I o & 1
\end{array}\right]}{s}=\frac{C o+c I o}{s} \\
I^{*}=\frac{\left[\begin{array}{ccc}
1 & -1 & 0 \\
-c & 1 & C o \\
0 & 0 & I o
\end{array}\right]}{s}=I o \\
I^{*}>0
\end{gathered}
$$

No obstante, el problema matemático se resuelve a costa de la capacidad de explicación del modelo.

\section{El modelo K}

Las ideas de Kalecki pueden formalizarse con base a los siguientes supuestos: una economía cerrada y sin gobierno, competencia imperfecta, los trabajadores no ahorran y los capitalistas tienen planes de inversión a realizar cada periodo.

Sobre el supuesto de la competencia imperfecta, la teoría de Kalecki se aleja de la propuesta keynesiana y guarda una relación directa con la formación de precios al estilo de la Economía Política Marxista. Para Kalecki (1982), los precios se definen por los costos y un margen determinado por el poder de mercado de las empresas. Para evitar ambigüedades en los valores de $m$ y $n$, los coeficientes que reflejan el grado de monopolio, se emplea la formalización de Borges (1991), donde el precio corresponde a una media ponderada entre 
el precio deseado y el precio medio de la industria. La ecuación de precios de Borges refleja que los aumentos en el grado de monopolio $(g)$ inciden positivamente en los precios $(p)$.

$$
\begin{gathered}
p=m * u+n\left(\bar{p}-m^{*} u\right) \\
p=m * u+n \bar{p}-m * u n \\
p=m^{*} u(1-n)+n \bar{p} \\
p=g u+n p \text { donde } g=m *(1-n)
\end{gathered}
$$

Marx (2012) define los precios en dos secciones. La parte interna corresponde a la empresa o el precio de costo $(p c)$ y la parte externa que establece la competencia o la tasa media de ganancia $(f)$.

$$
\begin{gathered}
p=p c+p c * g^{\prime} \\
p=p c\left(1+g^{\prime}\right) \\
p=p c^{*} f \text { donde } f=(1+g)
\end{gathered}
$$

En los dos autores la formación de precios toma como base el elemento que puede controlar la empresa, los costos, y el elemento de la competencia que define el poder para fijar un mayor precio.

El modelo K está compuesto por tres ecuaciones. La primera es la función de inversión $(I)$, donde la tasa de decisiones de inversión guarda una relación directa tanto con la tasa de ahorro $(S o)$ como con las ganancias $(G)$ y, por otro lado, mantiene una relación indirecta con la variación del acervo de capital $(K)$.

En la segunda ecuación las ganancias están determinadas por el consumo y por la inversión. Finalmente, el acervo de capital depende de una parte autónoma (Ko) y de una sensibilidad negativa con la inversión $(-d I)$. Donde el signo negativo indica que si el capital aumenta, se reduce la inversión y viceversa.

$$
\begin{gathered}
I=S o+a G-b K \\
G=C o+c I \\
K=K o-d l
\end{gathered}
$$

$\mathrm{Al}$ acomodar las variables y representando el sistema de la forma $A x=d$, se calculó el determinante de la matriz de coeficientes, cuyo resultado diferente de cero aseguró la solución trivial.

$$
\begin{gathered}
{\left[\begin{array}{ccc}
1 & -a & b \\
-c & 1 & 0 \\
d & 0 & 1
\end{array}\right]\left[\begin{array}{l}
I \\
G \\
K
\end{array}\right]=\left[\begin{array}{l}
S o \\
C o \\
K o
\end{array}\right]} \\
|A|=1-a c-b d
\end{gathered}
$$


Por lo tanto, las soluciones de equilibrio $\left(I^{*}, G^{*}, K^{*}\right)$ se determinaron con la regla de Cramer asegundando valores positivos para las tres variables del modelo $\mathrm{K}$ :

$$
I^{*}=\frac{\left[\begin{array}{ccc}
S o & -a & b \\
C o & 1 & 0 \\
K o & 0 & 1
\end{array}\right]}{1-a c-b d}=\frac{S 0-a C o-b K o}{1-a c-b d}
$$

$$
\begin{gathered}
G^{*}=\frac{\left[\begin{array}{ccc}
1 & S o & b \\
-c & C o & 0 \\
d & K o & 1
\end{array}\right]}{1-a c-b d}=\frac{c(S o-b K o)+C o(1 * b d)}{1-a c-b d} \\
K^{*}=\frac{\left[\begin{array}{ccc}
* & \\
1 & -a & S o \\
-c & 1 & C o \\
d & 0 & K o
\end{array}\right]}{1-a c-b c}=\frac{a(-d C o-c K o)-d S o+K o}{1-a c-b d}
\end{gathered}
$$

Si se compara el modelo keynesiano con el modelo K, se aprecia que la segunda propuesta tiene una mayor capacidad de explicar la inversión productiva. En términos de teoría económica, el modelo K explica la formación de precios y hablar de precios lleva a entender la determinación del margen y las decisiones de inversión, dentro de un esquema de incertidumbre (Ocampo, 1988).

La propuesta matemática asume que los fondos con los que se financia la inversión provienen de la acumulación interna. El modelo K no es reduccionista, no considera los movimientos de la tasa de interés ni somete la inversión a una variable exógena.

En última instancia, las ideas de Kalecki fueron el punto de partida para la teoría postkeynesiana, donde se asume que las ganancias permiten a las empresas autofinanciarse (Lavoie, 2004). ¿Por qué? En la realidad, las empresas se enfrentan a restricciones de recursos, los economistas heterodoxos retoman el principio del riesgo creciente de Kalecki (1982). El principio está basado en la incertidumbre, ya que conseguir dinero externo enfrenta a la 
empresa a un mayor nivel de endeudamiento; corriendo el riesgo de que generar un nivel inadecuado de flujo de efectivo y caer en insolvencia. De ahí que las empresas deben buscar otra forma de obtener recursos como autofinanciarse o moviendo el precio de las acciones.

\section{Un modelo kaleckiano con financiarización}

Dallery (2010) plantea que actualmente la dinámica económica ha cambiado por la penetración del sector financiero, es decir, por la presencia de la financiarización. Este fenómeno ha sido estudiado por varios autores y en diferentes dimensiones. Uno de ellos es Mateo (2015), quien hace un recuento con relación a las crisis y explica que no existe una definición única de este proceso económico (Cuadro 1).

En concreto, para una serie de autores con bases marxistas la financiarización se basa en un nuevo régimen de acumulación basado en el dominio de las finanzas (Bruno et al., 2011).

¿Cómo puede formalizarse un proceso global? Se toma como base los estudios de Stockhammer et al. (2011) y Mateo (2015) para medir el grado de financiarización ya que el indicador se relaciona con el endeudamiento de los hogares. Además, este indicador coincide con las variables del modelo K para poder explicar la dinámica de largo plazo de la inversión productiva.

\section{Cuadro 1}

Indicadores que miden Financiarización

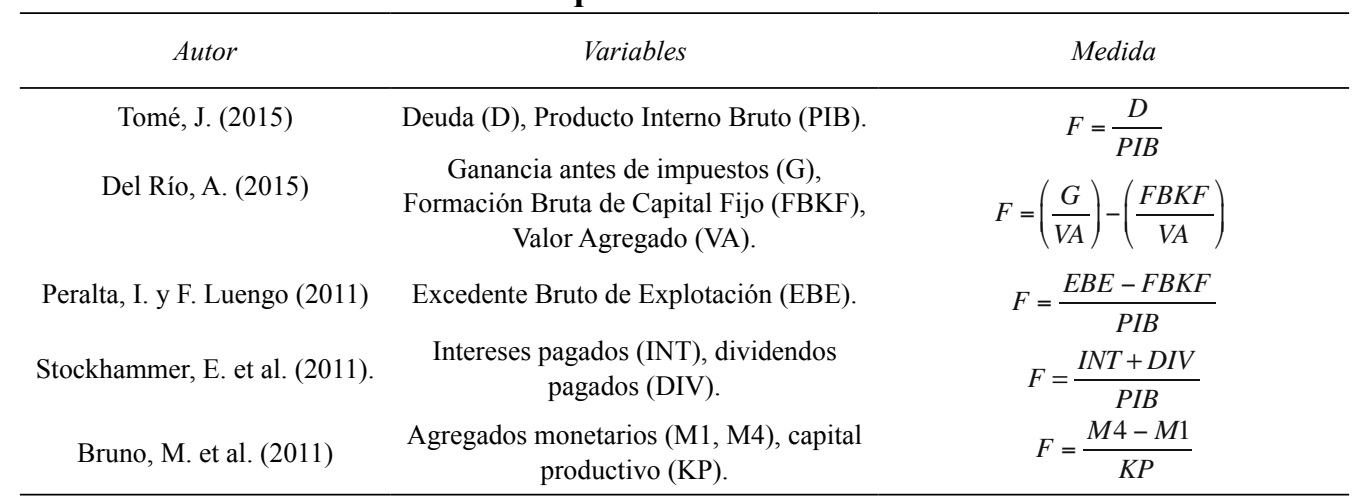

Entonces, el modelo asume cinco supuestos: la economía es imperfecta o existe un alto grado de monopolio, la economía es abierta y pequeña. Por ejemplo, un país sin gran participación comercial en el mundo, pero con flujos de capital importantes. Tercer supuesto, la economía está financiarizada. El grado de financiarización $(\pi)$ se mide con la proporción deuda a ingreso nacional: 


$$
\pi=\frac{D}{P I B}
$$

Como cuarto supuesto el consumo es exógeno y la propensión marginal a consumir es mayor a uno, es decir, el consumo se financia con crédito pues la clase trabajadora no existe ahorro. En otros términos, el crédito permite sostener el consumo mientras el salario se estanca.

$$
c>1 \quad s=0
$$

Finalmente, el modelo muestra cómo afecta la financiarización a la inversión y al ingreso. El cambio de los instrumentos de deuda reduce la inversión y el cambio de la tasa de finan74 ciarización afecta positivamente al PIB o es un modelo guiado por las ganancias (a diferencia de un régimen guiado por los salarios).

$$
\frac{d I}{d \pi o}<0 \quad \frac{d Y^{*}}{d \pi o}>0
$$

Entonces, se proponen tres ecuaciones para explicar las variables endógenas: inversión $(I)$, ingreso $(Y)$ y financiarización () .

$$
\begin{gathered}
I=S o+a Y-b \pi \\
Y=C o+c I+d \pi \\
\pi=\pi o-f I
\end{gathered}
$$

La inversión está integrada por un componente constante del ahorro y por una sensibilidad que mide el cambio en el ingreso. Además, se incorporó la relación negativa entre la financiarización y la inversión productiva.

El consumo autónomo, la inversión y la tasa de financiarización determinan el nivel del ingreso nacional, de donde emerge una relación directa entre el ingreso y la tasa de financiarización.

Por último, la tasa de financiarización se explica por un componente exógeno y por la relación indirecta con la inversión, de ahí que si aumenta el flujo de instrumentos de deuda se reducen los recursos que se destinan a la producción.

Para resolver el modelo se expresó matricialmente el sistema de ecuaciones de la forma $A x=d$ y se calculó su determinante: 


$$
\begin{gathered}
{\left[\begin{array}{ccc}
1 & -a & b \\
-c & 1 & -d \\
f & 0 & 1
\end{array}\right]\left[\begin{array}{c}
I \\
Y \\
\pi
\end{array}\right]=\left[\begin{array}{c}
S o \\
C o \\
\pi o
\end{array}\right]} \\
|A|=1-b f+a(d f-c) \\
|A|>0 s i(a c+b f-a d f)<1
\end{gathered}
$$

Las soluciones de equilibrio se hallaron con la regla de Cramer, porque el sistema tiene una solución trivial:

$$
\begin{gathered}
I^{*}=\frac{\left[\begin{array}{ccc}
S o & -a & b \\
C o & 1 & -d \\
\pi o & 0 & 1
\end{array}\right]}{1-b f+a(d f-c)}=\frac{\pi o(a d-b)+S o+a C o}{1-b f+a(d f-c)} \\
Y^{*}=\frac{\left[\begin{array}{ccc}
1 & S o & b \\
-c & C o & -d \\
f & \pi o & 1
\end{array}\right]}{1-b f+a(d f-c)}=\frac{C o(1-b f)+\pi o(d-b c)+S o(c-d f)}{1-b f+a(d f-c)} \\
\pi^{*}=\frac{\left[\begin{array}{ccc}
1 & -a & S o \\
-c & 1 & C o \\
f & 0 & \pi o
\end{array}\right]}{1-b f+a(d f-c)}=\frac{f(-a C o-S o)+\pi o(1-a c)}{1-b f+a(d f-c)} \\
Y^{*}>0 \\
\pi^{*}>0
\end{gathered}
$$

El efecto desplazamiento, cuando la financiarización reduce la inversión productiva, se materializa con la derivada de la inversión respecto al componente exógeno de la financiarización. Para que tenga sentido económico, la derivada debe ser menor a cero si

$$
\begin{gathered}
\frac{d I^{*}}{d \pi o}=\frac{a d-b}{1-b f+a(d f-c)} \\
\frac{d I^{*}}{d \pi o}<0
\end{gathered}
$$


La forma en que se organiza el capitalismo actualmente es un patrón guiado por las ganancias (Stockhammer, et al., 2011), es decir, cuando aumenta la tasa de financiarización se incrementa el ingreso.

$$
\begin{gathered}
\frac{d Y^{*}}{d \pi o}=\frac{d-b c}{1-b f+a(d f-c)} \\
\frac{d Y^{*}}{d \pi o}<0
\end{gathered}
$$

Matemáticamente es la derivada del ingreso respecto al componente autónomo de la financiarización, pero con la condición para que se cumpla la teoría.

\section{Conclusiones}

A lo largo de este trabajo se han estudiado varios planteamientos matemáticos con el fin de caracterizar las decisiones de inversión en la economía, desde la perspectiva productiva y financiera e incluyendo el fenómeno llamado financiarización.

El primer planteamiento es keynesiano convencional. Bajo el marco analítico del modelo IS-LM, se consideró que la inversión depende de la tasa de interés y ello tiene como resultado que las ecuaciones son inconsistentes matemáticamente. Entonces, se propuso que la inversión sea exógena para que el sistema de ecuaciones manifieste una solución trivial. Aunque se resuelve el problema matemático, se pierde capacidad de explicación.

En segundo lugar, el modelo kaleckiano K explica la tasa de decisiones de inversión con la tasa de ahorro, las ganancias y la variación del acervo de capital. La variable fundamental en la economía kaleckiana son las ganancias, ya que son los incentivos que guían a los inversionistas a expandir la capacidad productiva.

El modelo $\mathrm{K}$ no es reduccionista, no considera los movimientos de la tasa de interés ni somete la inversión a una variable exógena. Además, Kalecki inicia su análisis asumiendo una economía imperfecta, a diferencia de la propuesta keynesiana. Por estas dos razones, las ideas del autor polaco fueron el punto de partida para la teoría postkeynesiana.

En tercer lugar, sobre la base del modelo de Kalecki se ha planteado formalizar el proceso de financiarización que sufren las economías a lo largo de los últimos 30 años. El concepto no es homogéneo ni único, pero este trabajo acogió las ideas de Stockhammer, donde el grado de financiarización se mide con la proporción deuda a ingreso nacional y en esta propuesta matemática sobresalen dos elementos: un efecto desplazamiento ya que se incrementan los activos financieros a costa de la inversión productiva y se caracteriza el patrón guiado por las ganancias pues si aumenta la tasa de financiarización se incrementa el ingreso en la economía. 


\section{Referencias}

Borges, A. (1991). "Uma interpretação da equação de preços de Kalecki”, Revista de Economia Política, vol. 11, núm. 2, pp. 18-25.

Bruno, M. et al. (2011). "Finance-Led Growth Regime no Brasil: estatuto teórico, evidências empíricas e consequências macroeconômicas", Revista de Economia Política, vol. 31, núm. 5, pp. 730-750.

Dallery, T. (2010). "Post-Keynesian Theories of the Firm under Financialization", Review of Radical Political Economics, vol. 41, núm. 4, pp. 492-515.

Del Río, A. (2015). "La financiarización a debate: la desvinculación entre beneficios e inversión en el caso español, Investigación Económica, vol. 74, núm. 291, pp. 53-77.

Kalecki, M. (1982). Ensayos escogidos sobre la dinámica de la economía capitalista, México: Fondo de Cultura Económica.

Keynes, J. M. (2014). Teoría general de la ocupación, el interés y el dinero, México: Fondo de Cultura Económica.

Lavoie, M. (2004). La economía postkeynesiana. Un antídoto del pensamiento único, Barcelona: Icaria Editorial.

Marx, C. (2012). El capital III. Critica de la economía politica, México: Fondo de Cultura Económica.

Mateo, J. (2015). "La financiarización como teoría de la crisis en perspectiva histórica", Cuadernos de Economía, vol. 34, núm. 64, pp. 23-44.

Ocampo, J. (1988). Lecturas del Trimestre Económico, México: Fondo de Cultura Económica.

Peralta, I y F. Luengo (2011). "Financiarización, acumulación de capital y crecimiento salarial en la UE-15”, Investigación Económica, vol. 70, núm. 275, pp. 125-162.

Stockhammer, E. et al. (2011). "Financialization, income distribution, and aggregate demand in the USA”, Cambridge Journal of Economics, vol. 35, núm. 4, pp. 637-661. 